\title{
A note on repeated degrees of line graphs
}

\author{
Shimon Kogan \\ Department of Computer Science and Applied Mathematics \\ Weizmann Institue, Rehovot 76100, Israel \\ shimon.kogan@weizmann.ac.il
}

Submitted: May 24, 2020; Accepted: Feb 1, 2021; Published: Jul 30, 2021

(C) The author. Released under the CC BY-ND license (International 4.0).

\begin{abstract}
Let $\operatorname{rep}(G)$ be the maximum multiplicity of a vertex degree in graph $G$. It was proven in Caro and West [E-JC, 2009] that if $G$ is an $n$-vertex line graph, then $\operatorname{rep}(G) \geqslant \frac{1}{4} n^{1 / 3}$. In this note we prove that for infinitely many $n$ there is a $n$-vertex line graph $G$ such that $\operatorname{rep}(G) \leqslant(2 n)^{1 / 3}$, thus showing that the bound above is asymptotically tight. Previously it was only known that for infinitely many $n$ there is a $n$-vertex line graph $G$ such that $\operatorname{rep}(G) \leqslant \sqrt{4 n / 3}$ (Caro and West [E-JC, 2009]). Finally we prove that if $G$ is a $n$-vertex line graph, then $\operatorname{rep}(G) \geqslant\left(\left(\frac{1}{2}-o(1)\right) n\right)^{1 / 3}$.
\end{abstract}

Mathematics Subject Classifications: $05 \mathrm{C} 07$

\section{Introduction}

It is well-known that any graph with at least two vertices (no loops or multiedges) has two vertices with the same degree. We define the repetition number of a graph $G$, written $\operatorname{rep}(G)$, to be the maximum multiplicity in the degree sequence of $G$. For general graphs it was shown in [CW09] that $\operatorname{rep}(G) \geqslant n /(2 d-2 s+1)$ when $G$ has $n$ vertices, average degree $d$ and minimum degree $s$. Furthermore it was proven in [CW09] that the bound above is sharp for certain families of graphs. Several special graph families were considered in [CW09] besides their results on general graphs. We focus in this note on the natural question of the repetition number of line graphs. We denote by $L(G)$ the line graph of graph $G$. The following lower and upper bounds on the repetition number of line graphs were proven in [CW09].

Theorem 1.1. If $G$ is a graph with $m$ edges, then $\operatorname{rep}(L(G)) \geqslant \frac{1}{4} m^{1 / 3}$.

Theorem 1.2. For infinitely many $m$ there is a graph $G$ with $m$ edges and $\operatorname{rep}(L(G)) \leqslant$ $\sqrt{4 m / 3}$.

In fact the following stronger result is shown in [CW09]. 
Theorem 1.3. For infinitely many $m$ there is a tree $G$ with $m$ edges and $\operatorname{rep}(L(G)) \leqslant$ $\sqrt{4 m / 3}$. Furthermore this bound is asymptotically tight (for trees).

Conjecture 3.5 in [CW09] states that the minimum of $\operatorname{rep}(L(G))$ over $m$-edge graphs is $\Theta(\sqrt{m})$. In this note we refute this conjecture, by showing that the bound in Theorem 1.1 is asymptotically tight. In particular we prove the following theorems.

Theorem 1.4. For infinitely many $m$ there is a graph $G$ with $m$ edges and

$$
\operatorname{rep}(L(G)) \leqslant(2 m)^{1 / 3} \text {. }
$$

Theorem 1.5. If $G$ is a graph with $m$ edges, then

$$
\operatorname{rep}(L(G)) \geqslant\left(\left(\frac{1}{2}-o(1)\right) m\right)^{1 / 3} .
$$

The proof of Theorem 1.5 is very similar to the proof of Theorem 1.1, while the proof of Theorem 1.4 is completely different from the proof of Theorem 1.2. For more results on repeated degrees see [CSY14] and [CY20].

\section{Proof of Theorem 1.4}

Let $n=t^{2}$ be an integer. Let $G=(U, V, E)$ be a bipartite graph with $U=\left\{u_{1}, u_{2}, \ldots, u_{t}\right\}$ and $V=\left\{v_{1}, v_{2}, \ldots, v_{n}\right\}$. Furthermore we set the edges of $G$ in such manner that the following holds for the degrees of the vertices:

1. for all $1 \leqslant i \leqslant t$ we have

$$
d\left(u_{i}\right)=n-(i-1) t
$$

2. for all $1 \leqslant i \leqslant n$ we have

$$
d\left(v_{i}\right)=\left\lceil\frac{i}{t}\right\rceil
$$

Such degree sequence exists as we can set the edges of $G$ in the following manner. For each $1 \leqslant i \leqslant t$ set

$$
V_{i}=\left\{v_{1+(i-1) t}, \ldots, v_{t+(i-1) t}\right\}
$$

Now for each $1 \leqslant i \leqslant t$ we connect vertex $u_{i}$ to all the vertices in sets $V_{i}, \ldots, V_{t}$. The number of edges in graph $G$ is

$$
|E|=\sum_{i=1}^{t} d\left(u_{i}\right)=\sum_{i=1}^{n} d\left(v_{i}\right)=n \cdot \frac{t+1}{2}
$$

Definition 2.1. For an edge $e=(u, v) \in E$ define $w(e)=d(u)+d(v)$. A set $M=$ $\left\{e_{1}, e_{2}, \ldots, e_{r}\right\}$ of edges in $E$ is called homogeneous if for all $1 \leqslant i \leqslant j \leqslant r$ we have $w\left(e_{i}\right)=w\left(e_{j}\right)$. 
Notice that by definition we have $\operatorname{rep}(L(G))$ equal to the maximum cardinality of a homogeneous set, and hence to prove that $\operatorname{rep}(L(G)) \leqslant t$ it is sufficient to prove the following.

Theorem 2.1. For any homogeneous set $M$ we have $|M| \leqslant t$.

We will need a simple observation to prove Theorem 2.1.

Lemma 2.2. For any homogeneous set $M$, if $(u, v) \in M$ and $\left(u^{\prime}, v^{\prime}\right) \in M$ are two different edges such that $u, u^{\prime} \in U$ and $v, v^{\prime} \in V$, then $u=u^{\prime}$.

Proof. Assume by contradiction that $e_{1}=\left(u_{i}, v_{j}\right) \in M$ and $e_{2}=\left(u_{k}, v_{r}\right) \in M$ where $i \neq k$. Notice that by the definition of the degree sequence of graph $G$ we have

$$
n-(i-1) t+1 \leqslant w\left(e_{1}\right) \leqslant n-(i-2) t .
$$

Now if $k>i$ then

$$
w\left(e_{2}\right) \leqslant n-(i-1) t
$$

And if $k<i$ then

$$
w\left(e_{2}\right) \geqslant n-(i-2) t+1 .
$$

We got a contradiction to the fact that $w\left(e_{1}\right)=w\left(e_{2}\right)$ and thus we are done.

By Lemma 2.2 we have that the edges of a homogeneous set $M$ are incident to exactly one vertex in $U$ and hence $|M| \leqslant t$ as there are at most $t$ vertices of the same degree in $V$. This concludes the proof of Theorem 2.1.

We conclude that in graph $G$ we have

1. $|E| \geqslant \frac{1}{2} n^{3 / 2}$ ( this follows from Equation (2.1) ).

2. $\operatorname{rep}(L(G)) \leqslant \sqrt{n}$.

and this establishes Theorem 1.4.

\section{Proof of Theorem 1.5}

Let $E(G)$ be the set of edges of $G$. Recall that $|E(G)|=m$. Let $\Delta$ be the maximum degree of graph $G$. As before for each edge $e=(u, v) \in E(G)$ we define $w(e)=d(u)+d(v)$. Case 1: Assume that $\Delta \leqslant 2^{1 / 3} \cdot m^{2 / 3}$.

Let $S$ be the set of vertices in $G$ of degree at least $2 m^{7 / 12}$. Notice that $|S| \leqslant m^{5 / 12}$ as $2 m^{7 / 12} \cdot m^{5 / 12}=2 m$. Hence there are at most $\left(\begin{array}{c}m^{5 / 12} \\ 2\end{array}\right) \leqslant m^{5 / 6}$ edges in $G$ with both endpoints in $S$, that is $|E(G[S])| \leqslant m^{5 / 6}$. Thus the edge set $Q=E(G) \backslash E(G[S])$ satisfies $|Q| \geqslant m-m^{5 / 6}$ and for each edge $e \in Q$ we have $w(e) \leqslant \Delta+2 m^{7 / 12}$. Hence by the pigeonhole principle we have

$$
\operatorname{rep}(L(G)) \geqslant \frac{|Q|}{\max _{e \in Q} w(e)} \geqslant \frac{m-m^{5 / 6}}{2^{1 / 3} \cdot m^{2 / 3}+2 m^{7 / 12}} \geqslant\left(\left(\frac{1}{2}-o(1)\right) m\right)^{1 / 3} .
$$


and this concludes case 1 .

Case 2: Assume that $\Delta>2^{1 / 3} \cdot m^{2 / 3}$.

Let $v \in G$ be vertex of degree $\Delta$. Let $T$ be the set of neighbors of $v$ (in graph $G$ ) of degree at most $m^{5 / 12}$. As there are at most $2 m^{7 / 12}$ vertices of degree at least $m^{5 / 12}$ we have

$$
|T| \geqslant \Delta-2 m^{7 / 12} \geqslant(1-o(1)) 2^{1 / 3} \cdot m^{2 / 3} .
$$

Now we shall consider two subcases according to the number of edges in the subgraph of $G$ induced by $T$.

Subcase 2-1: Assume that $|E(G[T])| \geqslant 2 m^{9 / 12}$.

In this case we have by the pigeonhole principle that

$$
\operatorname{rep}(L(G)) \geqslant \frac{|E(G[T])|}{\max _{e \in E(G[T])} w(e)} \geqslant \frac{2 m^{9 / 12}}{2 m^{5 / 12}} \geqslant m^{1 / 3} .
$$

and this concludes subcase 2-1.

Subcase 2-2: Assume that $|E(G[T])|<2 m^{9 / 12}$. As $|E(G[T])|<2 m^{9 / 12}$ we have the following bound (where $d(u)$ is the degree of $u$ in graph $G)$.

$$
\sum_{u \in T} d(u) \leqslant|E(G)|+2 m^{9 / 12} \leqslant(1+o(1)) m .
$$

Now set $r=\operatorname{rep}(L(G))$. We may assume that

$$
r \leqslant m^{1 / 3}
$$

for otherwise we are done. Notice that in $T$ there are at most $r$ vertices of the same degree, as edges from $v$ to $T$ have a fixed endpoint, and thus any degree that occurs more than $r$ times in $T$ occurs more than $r$ times as a degree in $L(G)$. Hence we have the following inequality (we remind the reader that the $o(1)$ tends to 0 as $m$ tends to infinity).

$$
\begin{aligned}
\sum_{u \in T} d(u) & \geqslant r \cdot \sum_{i=1}^{\lfloor|T| / r\rfloor} i \\
& \geqslant r \cdot(\lfloor|T| / r\rfloor)^{2} / 2 \\
& \geqslant \frac{|T|^{2}}{2 r}\left(1-\frac{2 r}{|T|}\right) \\
& \geqslant \frac{|T|^{2}}{2 r}(1-o(1)) \\
& \geqslant \frac{m^{4 / 3}}{r 2^{1 / 3}}(1-o(1))
\end{aligned}
$$

Now combining Inequalities (3.4) and (3.2) we get

$$
\frac{m^{4 / 3}}{r 2^{1 / 3}}(1-o(1)) \leqslant(1+o(1)) m .
$$

We conclude that $r \geqslant\left(\frac{m}{2}\right)^{1 / 3}(1-o(1))$ and the theorem follows. 


\section{Acknowledgements}

Work supported in part by the Israel Science Foundation (grant No. 1388/16).

\section{References}

[CW09] Yair Caro and Douglas B. West. Repetition number of graphs. The Electronic Journal of Combinatorics, 16, \#R7, 2009.

[CSY14] Yair Caro, Asaf Shapira, and Raphael Yuster. Forcing $k$-repetitions in degree sequences. The Electronic Journal of Combinatorics, 21(1):\#P1.24, 2014.

[CY20] Yair Caro and Raphael Yuster. Induced subgraphs with many repeated degrees. Discrete Mathematics, 343(5):111828, 2020. 